\title{
Keratoglobus: a close entity to megalophthalmos
}

\author{
Noopur Gupta and Anita Ganger
}

\begin{abstract}
Background: Keratoglobus closely resembles buphthalmos and anterior megalophthalmos.

Findings: A 45-year-old man presented with gradually progressive, painless, diminution of vision in both eyes since childhood. On examination, visual acuity of right (RE) and left eye (LE) was 20/60 and 2/20 respectively. Clinical pictures of the patient are shown in panel A, B, C, D. Keratometry values were $46.47 / 47.94 \mathrm{D}$ at $42 / 132^{\circ}$ in RE and 46.90/47.23 D at 174/84 in LE, signifying steep, ectatic cornea. Axial lengths, anterior chamber depth and corneal thickness in RE/LE was 23.53/27.12 mm, 5.18/4.48 mm and 413/420 $\mu \mathrm{m}$ respectively. Iridodonesis was noted in left eye. Retinal evaluation of LE revealed retinal detachment (RD) with posterior staphyloma due to high myopia, hereas RE was within normal limits. Intraocular pressure was normal in both eyes. Final diagnosis was keratoglobus with LE myopic RD. The patient improved to 20/30 in right eye with no improvement in LE with scleral contact lens.
\end{abstract}

Conclusion: Keratoglobus, Megalophthalmos and Buphthalmos are exceedingly close entities and it is very essential to make correct diagnosis, as management options differ significantly for all three diseases.

A 45-year-old male patient presented with gradual, progressive and painless diminution of vision since childhood. On ocular examination, visual acuity of right eye (RE) and left eye (LE) was $20 / 60$ and $2 / 20$ respectively. Slit lamp evaluation revealed bilateral, diffuse corneal thinning in $\mathrm{BE}$ with outward, globular protrusion of the cornea (shown in panel A, B, C, D). The intraocular pressure (IOP) recorded with applanation tonometer was in the normal range. Keratometric values, depicting the corneal curvature, were $46.47 / 47.94$ dioptre (D) at $42 / 132^{\circ}$ and $46.90 / 47.23 \mathrm{D}$ at $174 / 84^{\circ}$ in $\mathrm{RE}$ and LE respectively, indicating steep and ectatic cornea bilaterally. Corneal topography assessed by Pentacam Scheimpflug Imaging system revealed, sim $\mathrm{K}$ values of 46.70/47.32 (steep axis at $60.9^{\circ}$ ) in $\mathrm{RE}$ and $45.9 / 47.7$ (steep axis at $172.9^{\circ}$ ) in $\mathrm{LE}$ along with a posterior elevation of $+12 /+25$ in $\mathrm{RE} / \mathrm{LE}$ respectively. The recorded values of white-to-white diameter, axial length, corneal thickness and anterior chamber depth in RE/LE were 14.59/14.15 mm, 23.53/27.12 mm,

\footnotetext{
*Correspondence: anitaganger@gmail.com

Cornea and Refractive Surgery Services, Dr. Rajendra Prasad Centre

for Ophthalmic Sciences, All India Institute of Medical Sciences,

New Delhi 110029, India
}

413/420 $\mu \mathrm{m}$ and 5.18/4.48 mm respectively. Retinal evaluation of LE revealed retinal detachment with posterior staphyloma, whereas RE was unremarkable. A diagnosis of bilateral keratoglobus with myopic retinal detachment in the LE was made. The risk of corneal perforation, even on minimal trauma was explained to the patient. On refraction no improvement in visual acuity was noted. Furthermore, the patient improved to 20/30 in the RE with a scleral contact lens while no improvement was noted in LE. The patient wilfully gave his consent to publish his medical details and images in a medical journal (Fig. 1).

\section{Comment}

Keratoglobus is a bilateral, non-inflammatory, ectatic disorder of the cornea that is characterized by globular protrusion of the cornea (Smolek and Klyce 2000). Interestingly, this disease closely resembles buphthalmos and anterior megalophthalmos, where abnormal, large eyes with enlarged cornea as well as increased axial lengths are seen in the presence and absence of glaucoma respectively (Table 1) (Dua et al. 1999). Although our patient presented with features, indicative of megalophthalmos i.e. presence of enlarged 

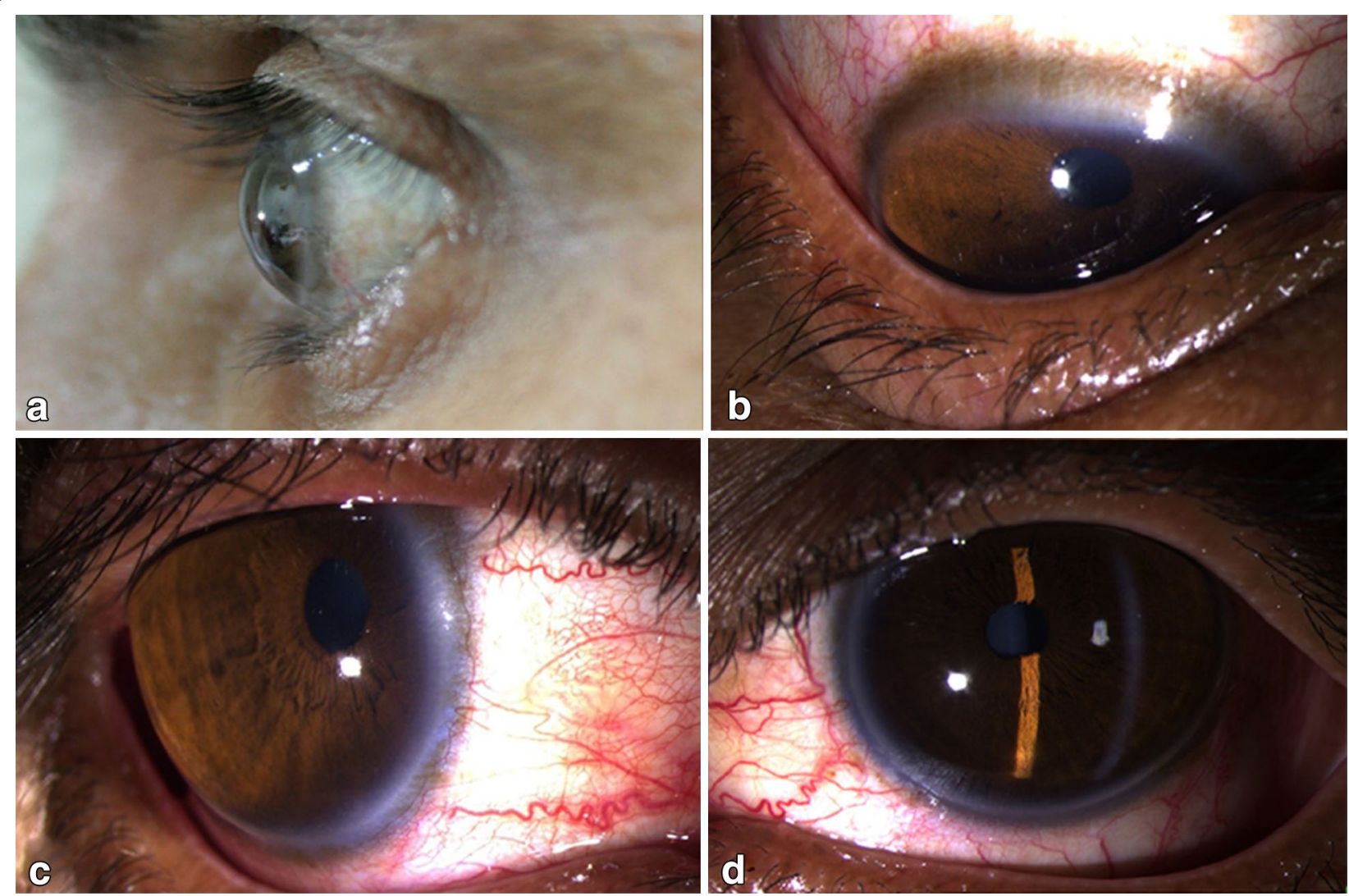

Fig. 1 a Clinical picture of the patient showing diffuse thinning of cornea with outward globular protrusion. $\mathbf{b}$ Indentation of lower lids is shown on downward gaze, due to the protruded cornea. $\mathbf{c}$, d Clinical pictures of the patient, showing bilateral clear cornea with deep anterior chamber with and stretched-out limbus

Table 1 Enumeration of differentiating points between keratoglobus, anterior megalophthalmos and buphthalmos

\begin{tabular}{llll}
\hline & Keratoglobus & Anterior megalophthalmos & Buphthalmos/infantile glaucoma \\
\hline Inheritance & No definite pattern & X-linked recessive & Sporadic \\
Age of presentation & Puberty & Congenital & First year of life \\
Natural History & Progressive & Non-progressive & Progressive \\
Symptoms & Frequent change of glasses & Variable and nonspecific & Watering, photophobia \\
Intra ocular pressure (IOP) & Normal & Normal & Elevated \\
Corneal diameter & $>13 \mathrm{~mm}$; symmetric with increased & $>13 \mathrm{~mm}$; symmetric & Variable, depends upon severity of \\
& WTW diameter & & glaucoma \\
Axial length & Increased axial length & Normal & Increased axial length \\
Iris & Iridodonesis \pm & Iridodonesis; iris stromal hypoplasia & Normal with high insertion \\
Optic disc & Usually normal & Normal with increased propensity for & Cup:disc ratio increased \\
& Refractive error correction and kerato- & Refractive errors correction & Control of IOP (medically/surgically) \\
Treatment & plasty in advanced cases & & \\
\end{tabular}

corneas, increased axial lengths, iridodonesis and absence of increased IOP (Tsai et al. 2005), but in view of steep corneal curvatures, abnormal and thin cornea, increased white to white diameter (Lockington and
Ramaesh 2015), normal intraocular pressure, absence of both miosis and ciliary ring enlargement, a diagnosis of buphthalmos or anterior megalophthalmos was excluded. 


\section{Conclusion}

Keratoglobus, Megalophthalmos and Buphthalmos are exceedingly close entities and it is very essential to make a correct diagnosis, as management options differ significantly for all three diseases (Table 1).

\section{Authors' contributions}

Both the authors, mentioned for this manuscript contributed considerably in clinical evaluation of the patient and manuscript writing. Both authors read and approved the final manuscript.

\section{Acknowledgements}

The authors acknowledge the experts from the Cornea and Refractive Surgery Services, Dr. Rajendra Prasad Centre for Ophthalmic Sciences, AllMS, New

Delhi for their guidance.

\section{Competing interests}

The authors declare that they have no competing interests.
Received: 17 March 2016 Accepted: 9 May 2016

Published online: 17 May 2016

\section{References}

Dua HS, Azuara-Blanco A, Pillai CT (1999) Cataract extraction and intraocular lens implantation in anterior megalophthalmos. J Cataract Refract Surg 25:716-719

Lockington D, Ramaesh K (2015) Use of a novel lamellar keratoplasty with pleat technique to address the abnormal white-to-white diameter in keratoglobus. Cornea 34:239-242

Smolek MK, Klyce SD (2000) Is keratoconus a true ectasia? An evaluation of corneal surface area. Arch Ophthalmol 118:1179-1186

Tsai C-K, Lai I-C, Kuo H-K, Teng M-C, Fang P-C (2005) Anterior megalophthalmos. Med J 28:191-195

\section{Submit your manuscript to a SpringerOpen ${ }^{\odot}$ journal and benefit from:}

- Convenient online submission

- Rigorous peer review

Immediate publication on acceptance

- Open access: articles freely available online

- High visibility within the field

- Retaining the copyright to your article 